\title{
Análisis del estado de arte de Técnicas de Soft Computing aplicadas a problemas de planificación de red en $5 \mathrm{G}$.
}

\author{
Analysis of the state of the art of Soft Computing Techniques applied to network \\ planning problems in $5 G$.
}

Vasconez Núñez Vanessa Alexandra. ${ }^{1}$

Recibido: 15-03-2020 / Revisado: 21-04-2020 /Aceptado: 09-05-2020/ Publicado: 05-06-2020

\begin{abstract}
.
DOI: $\underline{\text { https://doi.org/10.33262/concienciadigital.v3i2.2.1246 }}$
\end{abstract}

The current article developed a review of the state of the art about the application of Soft Computing techniques in solving planning problems of $5 \mathrm{G}$ networks. To achieve this, the different existing Soft Computing techniques were classified (neural networks, fuzzy logic, evolutionary algorithms) and models proposed and methods of solution were considered from works and research developed about the subject according to their authors. Additionally, the most relevant investigations described techniques which are specified to solve problems of architectures and crucial functionalities in the development of this technology, highlighting the following: finding an optimal position for a Base Station (BS) in an area of particular interest, operating in the desired multiple frequency bands while maintaining high gain, limiting power consumption in $5 \mathrm{G}$ network infrastructures, and trying to increase quality of service by decreasing the probability of call blocking. To conclude, the most applied Soft Computing techniques in solving planning problems of 5G networks are fuzzy logic, in order to limit the energy consumption in 5G network infrastructures, in addition to artificial neural networks and genetic algorithms for admission of calls in 5G networks, increasing quality of service by reducing interference.

Key Words: Soft Computing, Planning, 5G Networks, Communications, Interference.

\section{Resumen.}

En el presente artículo se realizó una revisión del estado del arte de la aplicación de las técnicas de Soft Computing en la resolución de problemas de planificación de redes 5G, para

\footnotetext{
${ }^{1}$ Universidad Nacional de Chimborazo, Chimborazo, Ecuador, vavasconez@unach.edu.ec
} 
lo cual se clasificó las diferentes técnicas de soft computing existentes (redes neuronales, lógica difusa, algoritmos evolutivos) y de los trabajos e investigaciones realizados sobre el tema según sus autores, los modelos planteados y los métodos de solución. Adicionalmente se describieron las investigaciones más relevantes en donde se especifican técnicas para dar solución a los problemas de arquitecturas y funcionalidades cruciales en el desarrollo de esta tecnología, entre los cuales se resalta: encontrar una posición óptima para una Estación Base (BS) en un área de interés determinada, operar en las bandas de frecuencias múltiples deseadas mientras se mantiene una alta ganancia, limitar el consumo de energía en las infraestructuras de red 5G y tratar de incrementar la calidad del servicio al disminuir la probabilidad de bloqueo de llamadas. Finalmente se concluyó que las técnicas de soft computing más aplicadas a la solución de problemas de planificación de las redes 5G son lógica difusa, para limitar el consumo de energía en las infraestructuras de red 5G, además de las redes neuronales artificiales y algoritmos genéticos para la admisión de llamadas en redes $5 \mathrm{G}$ con la finalidad de incrementar la calidad del servicio al disminuir las interferencias.

Palabras claves: Soft_computing, Planificación, Redes_5G, Comunicaciones, Interferencia.

\section{Introducción.}

Desde la aparición de las comunicaciones en el mundo, estas han presentado cambios acorde a las necesidades de las generaciones, originando nuevas formas de evolución de las telecomunicaciones, produciendo cambios en los paradigmas actuales; en virtud a ello se han desarrollado de forma paralela innovaciones tanto en hardware como en software de los componentes que intervienen en estas tecnologías (González \& Salamanca, 2016), pues como se indica en (Maldonado, 2013), el ser humano es capaz de realizar diversas tareas inmerso en el mundo de incertidumbre e imprecisión en el que vive, tareas como la comprensión de un mensaje no carente de ruido, el reconocimiento o clasificaciòn de imágenes o la conducción de vehículos, entre otras.

En relación se añade que el desarrollo del software actualmente va cambiando las prácticas de la sociedad, pues se encuentra presente en diversas áreas de interés de los individuos, entre ellas se menciona la ingeniería, la industria, la medicina, el campo científico, gubernamental y de las empresas en general (Crespo, Peña, Pascual, \& Fustiel, 2016).

En este sentido en (Martín, 2015) se añade que actualmente existen una multitud de métodos, herramientas y metodologías que tratan de colaborar a la realización de esta labor, sin embargo, en la aparición de nuevos retos o tecnologías hace que cualquier planificación inicial deje de ser válida en poco tiempo.

La red 5G (quinta generación) es un conjunto de tecnologías creadas para proporcionar ganancias de altas velocidades de datos, sin embargo, estas ganancias se producen a expensas de una gran 
interferencia en la comunicación, es ahí donde se utilizan las técnicas de soft computing, las cuales se han propuesto resolver estos problemas, eliminando efectivamente la interferencia y en consecuencia mejorar la eficiencia hacia el usuario, adaptándose dinámicamente a las diferentes cargas de tráfico.

De esta manera se indica que las técnicas de soft computing buscan reemplazar a un humano para realizar una tarea de control o toma prestadas ideas de cómo los sistemas biológicos resuelven problemas y lo aplica a los procesos de control (Kumar \& Mahajan, 2009).

Esta tecnología es tolerante a la imprecisión, la incertidumbre, la verdad parcial para conseguir la trazabilidad, la aproximación, la robustez, el bajo costo de la solución y una mejor relación con la realidad, es por ello que el modelo a seguir para la informática suave es la mente humana (Kumar, Kumar, Das, \& Burnwal, 2013).

La búsqueda de soluciones para atender la creciente demanda de velocidad en redes móviles actuales, con velocidades más altas, mayores capacidades, mayor seguridad, menor consumo de energía y dar solución a las dificultades de implementación práctica establecen retos importantes que deben abordarse, dando lugar a diversos nichos de investigación, motivo por el cual en el presente artículo se realiza una revisión de la literatura de las técnicas de soft computing aplicadas a problemas de planificación de red en 5G, para identificar la realidad actual de las soluciones propuestas por diversos autores.

Para un mejor entendimiento de la investigación fue necesario la revisión de diversos conceptos y teorías referente al tema, entre los cuales se encuentran los siguientes:

\section{Soft computing}

El soft computing es una combinación sinérgica de metodologías de inteligencia artificial para modelar y resolver problemas del mundo real que son imposibles o demasiado difíciles de modelar matemáticamente, obteniendo soluciones robustas, manejables y con un coste menor que las que se obtienen utilizando técnicas convencionales (Atayero \& Luka, 2012).

Esta técnica es considerada como una herramienta para realizar diversas operaciones informáticas, centradas en el diseño de sistemas inteligentes que son capaces de manejar de forma adecuada la información incierta, imprecisa o incompleta, que incluyen redes neuronales, lógica difusa, modelos de razonamiento aproximado y algoritmos evolutivos como algoritmos genéticos y recocido simulado (Panda \& Patra, 2013).

\section{Redes Neuronales.}

Al-Maitah, Semenova, Semenov, Kulakov, \& Kucheruk (2018) mencionan que las redes neuronales consisten en neuronas artificiales inerconectadas, las cuales pueden adquirir, almacenar 
y emplear conocimiento experto, además de aprender nuevos patrones. Estas redes son aplicadas en la solución de problemas como la optimización, clasificación, coincidencia de patrones, proximación de funciones y agrupamiento de datos. De igual forma Atayero \& Luka (2012) se afirma que las redes neuronales representan un enfoque no algorítmico para el procesamiento de información y que se encuentran inspirados en los sistemas nerviosos biológicos; el procesamiento de la información sucede en muchos elementos de procesamientos simples llamados nodos, que son conocidos como células, unidades o neuronas. Cada enlace de conexión entre nodos se encuentra asociado con un peso, el cual controla e comportamiento de la red neuronal artificial entrenada. Las redes neuronales ayudan a compensar el costo computacional que se asocia con el complejo análisis matemático y electromagnético.

\section{Lógica Difusa}

Referente a la lógica difusa se indica que es una lógica multivaluada que permite representar de forma matemática la incertidumbre, otorgando herramientas formales para el tratamiento (González C. , 2011); mientras que Santos \& Miranda (2012) mencionan que "una de las características esenciales de la lógica difusa es que permite utilizar el lenguaje ordinario como lenguaje de descripción en un ordenador, incorporando así el conocimiento de un experto en una tarea determinada" (p. 103). Además la lógica difusa puede definirse como un sistema que modela funciones no lineales teniendo variables lingüísticas de entrada, las mismas convierte en variables de salida por medio de la lógica difusa y grupos de reglas como se puede visualizar en la figura siguiente (Angulo, Gil, \& Salcedo, 2017).

Figura 1. Esquema general de la lógica difusa

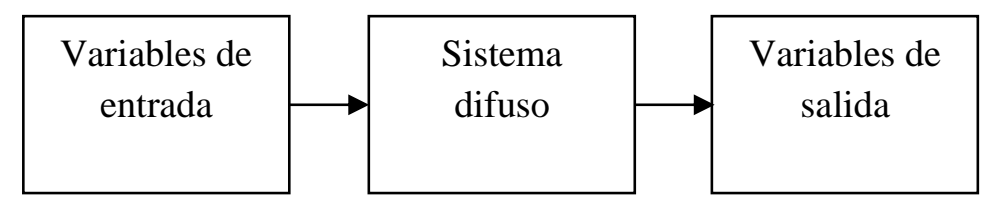

Fuente: (Angulo, Gil, \& Salcedo, 2017)

La lógica difusa se convierte en una propuesta para incorporar en los sistemas de ingeniería el conocimiento que se expresa en términos lingüísticos o expresiones verbales (Ramírez, Sarmiento, \& López, 2018), de acuerdo a este concepto se puede indicar que esta metodología permite definir valores en las áreas oscuras entre las evaluaciones convencionales de la lógica precisa como: Sí/No, Blanco/Nego, entre otras. Con la lógica difusa las proporciones se pueden representar con grados de certeza o falsedad (Angulo, Gil, \& Salcedo, 2017).

\section{Algoritmos evolutivos}

Los algoritmos evolutivos que son algoritmos matemáticos que convierte un conjunto de objetos matemáticos individuales en relación al tiempo, con la utilización de operaciones modeladas acorde 
al principio Darwiniano de supervivencia del más apto, posterior a haber presentado de manera natural un conjunto de operaciones genéticas (Castañeda, Garmendia, \& Santos, 2011). De igual forma el algoritmo evolutivo puede exporar todo un conjunto de valores y comparalos entre ellos, determinando de manera estocásticas cuál es superior entre ellos, esperando que esta respuesta sea cercana o igual al máximo general; sin embargo, los algoritmos evolutivos trabajan de manera diferente a otros mecanismos de optimización, puesto que al encontrarse basado en técnicas de selección natural, necesita un conjunto población, el mismo que debe representar múltiples puntos del espacio de búsqueda (Veslin, 2013); motivo por el cual Peñaranda (2015) se aporta que los algortimos genéticos resultan muy útiles cuando se tiene espacios de búsqueda amplios, complejos y muy poco explorados.

\section{Redes 5 G}

5G representan las siglas que se utilizan para referirse a la quinta generación de tecnologías de telefonía móvil, este conjunto de tecnologías representa la nueva revolución que transformará la industria de las telecomunicaciones, puesto que entre sus beneficios se encuentra una mayor velocidad de navegación y con una baja latencia, convirtiendo a los teléfonos móviles en dispositivos cada vez más eficientes en el aspecto energético (Fundacio Universitat Empresa, 2019).

Gracias a la quinta generación, se podrán conectar millones de dispositivos a internet, con una gran trasparencia de datos de manera inmediata, un ejemplo de ello es la transferencia de información con mayor rapidez entre el automóvil y el peatón, lo cual dará lugar al uso de vehículos autónomos (Fundacio Universitat Empresa, 2019), se obtendrá una banda ancha mejorada en entornos interiores y exteriores, realidad virtual y aumentada, además dará lugar a una agricultura inteligente, control energético, hogares inteligentes y seguimiento a distancia (Sanou, 2018).

Según menciona el (Ministerio de Tecnologías de la Información y las Comunicaciones de Colombia (2019), haciendo una analogía de la cadena de valor de la tecnología 5G en el ecosistema digital como se puede ver en la figura 2 , dentro de cada componente se puede encontrar los siguientes:

- Insumos: Dentro de los insumos esenciales para prestar servicios 5G se encuentra el espectro radioeléctrico, considerando que para la tecnología $5 \mathrm{G}$ se requiere un espectro en tres bandas que son: inferiores a $1 \mathrm{GHz}$, de 1-6 GHz y superiores a $6 \mathrm{GHz}$.

- Infraestructura: representan los componentes físicos de las redes por medio de los cuales se soportan los servicios 5G, encontrándose en este grupo las torres, cables, fibra óptica, entre otras.

- Servicios TIC: representan los servicios prestados por medio de la infraestructura 5G, incursionando servicios como Big Data, comunicación M2M, IoT, los cuales requerirán una alta disponibilidad y baja latencia como los vehículos autónomos. 
ISSN: 2600-5859

- Interfaz: Son los dispositivos que permiten a los usuarios acceder a los diferentes servicios tecnológicos que pueden ofrecer, tales como los teléfonos móviles, Pc, enrutadores, entre otros.

Figura 2. Ecosistema digital $5 \mathrm{G}$

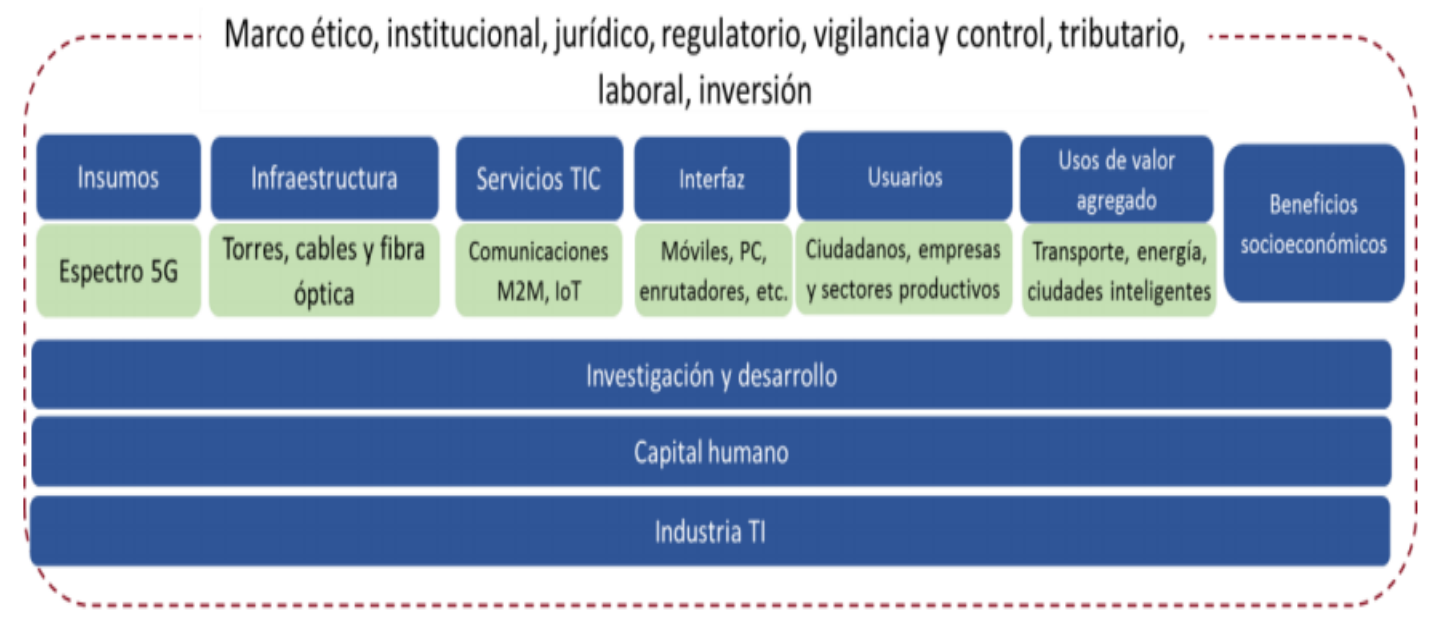

Fuente: (Ministerio de Tecnologías de la Información y las Comunicaciones de Colombia, 2019)

Usuarios: son las personas, entidades y sectores productivos que pueden acceder a los servicios que permitirá desplegar la tecnología 5G.

Usos del valor agregado: representan formas novedosas de utilizar los servicios 5G para facilitar los procesos y actividades, tales como el transporte, distribución de energía, ciudades inteligentes, entre otras.

Las grandes velocidades y la baja latencia que brinda la 5G impulsarán a las sociedades hacia la generación de ciudades inteligentes e internet de las cosas (IoT), es por ello que al hablar de 5G se debe referir a tres categorías importantes sobre sus posibles casos de uso, las mismas que se pueden observar en la figura 3.

Figura 3. Casos de uso de las redes 5G

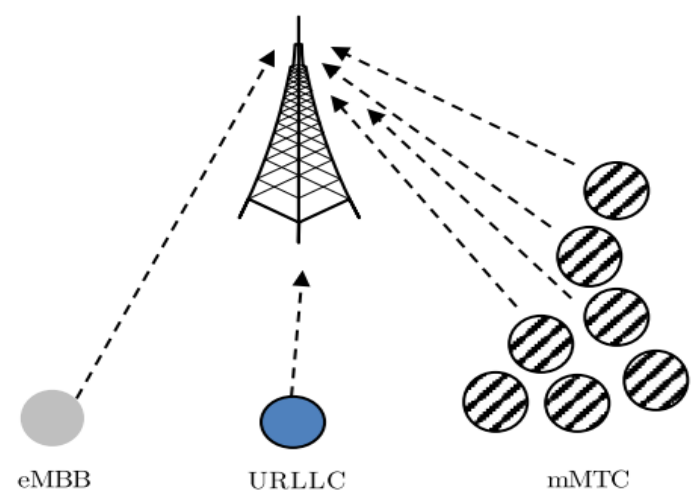

Fuente: (Popovski, Trillingsgaard, Kasper, Simeone, \& Durisi, 2018) 
Banda Ancha móvil mejorada (eMBB): es la banda ancha mejorada en entonos interiores y exteriores, además de la realidad virtual y aumentada, su objetivo es ofrecer un rendimiento máximo de hasta $10 \mathrm{Gbps}$, un rendimiento de $1 \mathrm{Gbps}$ en alta movilidad y un tráfico de red total de hasta 10,000x (Sanou, 2018).

Comunicaciones masivas entre máquinas (mMTC): un dispositivo mMTC está activo de forma intermitente y utiliza una velocidad de transmisión fija, generalmente baja, en el enlace ascendente. Se puede conectar una gran cantidad de dispositivos mMTC a una estación base (BS) determinada, pero en un momento dado solo un subconjunto desconocido (aleatorio) se activa e intenta enviar sus datos, que permite la administración de infraestructura, el monitoreo ambiental y las aplicaciones de atención médica. Por ejemplo, aplicaciones de teléfonos inteligentes para la integración de la asistencia sanitaria con dispositivos de fitness; tecnología vehicular, entre otras (Popovski, Trillingsgaard, Kasper, Simeone, \& Durisi, 2018).

Comunicaciones ultrafiables y de baja latencia (uRLLC): El soporte de transmisiones intermitentes de URLLC requiere una combinación de programación, para asegurar una cierta cantidad de previsibilidad en los recursos disponibles y, por lo tanto, soportar una alta confiabilidad; así como acceso aleatorio, para evitar que demasiados recursos estén inactivos debido al tráfico intermitente. Por ejemplo, acceso remoto que incluye desastres naturales, atención médica, comunicaciones militares, comunicación industrial, así como aplicaciones automotrices que requieren alta precisión (Popovski, Trillingsgaard, Kasper, Simeone, \& Durisi, 2018).

Con esta revisión se puede resaltar los grandes beneficios que ofrecerán las redes 5G, sin embargo también es importante resaltar que para su aplicación a la realidad se requiere una infraestructura adecuada para mejorar la conectividad en zonas rurales y poder extender su cobertura en todos los rincones, para lo cual es importante hacer uso de nuevas tecnologías y metodologías que proporciones soluciones rápidas y eficientes a la hora de su planificación e implementación.

\section{Metodologia.}

Con la finalidad de obtener una mejor comprensión del tema de investigación, se empleó una metodología basada principalmente en técnicas cualitativas para recolectar información.

\section{Métodos:}

Método analítico-sintético: Este método se refiere a dos procesos intelectuales que trabajan en unidad; el análisis que permite descomponer mentalmente un todo en sus partes y características, en varias relaciones y componentes y estudiar su comportamiento y la síntesis que establece mentalmente la combinación de las partes que se han analizado previamente y permite establecer relaciones o características generales entre los elementos de la realidad (Rodríguez \& Pérez, 2017). 
El método fue utilizado para analizar la documentación referente al objeto de estudio, lo cual permitió la extracción de los elementos más importantes que se relacionan con el mismo y de esa forma construir nuevos conocimientos partiendo de las experiencias previas.

Método de lectura científica: Este método ayudó a realizar una investigación descriptiva, ejecutando una interpretación crítica de las fuentes bibliográficas relacionadas con la temática específicamente de las técnicas de Soft Computing y su aplicación a problemas de planificación de red en 5G. Para la elaboración de este trabajo se han empleado bases de datos de: Dialnet, Scielo, IEEE, Springer, IJEAT.

\section{Criterios de inclusión}

Los principales criterios para selección de artículos son los que se mencionan a continuación:

- Publicaciones en inglés y español

- Artículos publicados en los últimos 10 años

- Artículos con acceso gratuito

\section{Criterios de exclusión}

Para descartar artículos se toma como criterios los siguientes parámetros:

- Artículos con acceso pagado al texto completo

- Artículos con una calidad baja una vez realizada la lectura crítica

- Artículos con fechas de publicación muy antiguas.

\section{Resultados.}

Actualmente existen diversos estudios acerca de la aplicación de las técnicas de soft computing en los sistemas de telecomunicaciones y en especial en la solución de problemas de implementación de las redes 5G. En relación a ello, Agredo, Jojoa, \& Almenar (2015) en su investigación denominada "Sistema MIMO con elevado número de antenas: clave 5G inalámbrica" determinan que contar con una gran cantidad de antenas en la estación base representa una solución a las necesidades de 5G, sin embargo en los algoritmos actuales que se han utilizado en Entradas Múltiples y Salidas Múltiples (MIMO) aparecen problemas de complejidad o desempeño cuando se trata de la utilización de un gran número de antenas por la complejidad en la detección de señales, lo cual se puede dar solución con la aplicación de técnicas basadas en otros algoritmos, metaheurísticas o en general, soft computing, algunas sub-óptimas pero de baja complejidad para hacer viable la implementación real de sistemas L-MIMO y en especial el aprovechamiento de sus beneficios.

Esta investigación se complementa con el trabajo denominado "Un estudio de diseño de antenas $5 \mathrm{G}$ optimizadas mediante algoritmos genéticos" en donde se presentó el diseño de tres estructuras 
de antena de interés para aplicaciones 5G. Los diseños de antena incluyeron: 1) parche pixelado, 2) parche con clavijas de cortocircuito y 3) antena monopolo. Cada uno de los diseños que se presentaron estuvieron planteados para operar en las bandas de frecuencias múltiples deseadas mientras se mantiene una alta ganancia. Estos diseños de antena se optimizan con la utilización de algoritmos genéticos (GA) y así lograr el rendimiento deseado, entre estos tres diseños de antena, la antena de parche pixelada proporcionó los mejores resultados y también es más simple de fabricar (Gjokaj, Doroshewitz, Nanzer, \& Chahal, 2017).

En relación a la eficiencia energética, Demestichas, Adamopoulou, \& Choras (2017) centró su estudio en la explotación del Protocolo de Control de Transmisión Multitrayecto (MPTCP), junto con técnicas de lógica difusa, para limitar el consumo de energía en las infraestructuras de red 5G. MPTCP se puede usar junto con los sistemas transmisor / receptor de entradas múltiples y salidas múltiples o por sí solo. El autor presentó la idea de acoplar el uso de MPTCP con una técnica de lógica difusa especialmente diseñada para el control de la transmisión. La técnica empleada es una adaptación del método de lógica difusa generalizada menos conocido, el mismo que fue llamado Números difusos ordenados (OFN), que permite la asociación del cambio de tendencia a un número difuso. De igual forma en la investigación denominda "Algoritmo genético con método de inteligencia de ubicación para la optimización de energía en redes inalámbricas 5G" se emplearon algoritmos evolutivos (AE) para resolver muchos problemas prácticos, como encontrar una posición óptima para una Estación Base (BS) en un área de interés determinada. El problema a resolver con la aplicación de esta técnica es que los usuarios se encuentran en áreas densas, llamadas áreas urbanas, y se requirió dar servicio a la mayor cantidad de usuarios posible utilizando la menor cantidad de BS y menos potencia de transmisión ubicando las BS en posiciones óptimas. Para resolver este problema, se aplicó el Algoritmo Genético Codificado Real (RGA) porque todas las variables de decisión (potencia de transmisión y ubicación) son valores continuos (Sachan, Jong Choi, \& Wook, 2016).

Además, Chabbouh, Ben, \& Choukair (2018), realizó una amplia investigación acerca del Cloud Radio Access Network (Cloud RAN) que es una de las arquitecturas móviles más prometedoras para redes 5G, lo cual consiste en separar las unidades de procesamiento de banda base digital (BBU) de los sitios celulares convencionales, de las Cabeceras de radio remotos (RRH), y virtualizar sus funciones en la nube para el procesamiento y la gestión centralizados de la señal. El mecanismo de agrupamiento propuesto tiene como objetivo reducir el consumo de energía de la red, sin comprometer la calidad de servicio (QoS) del usuario, es decir, el algoritmo propuesto redujo la complejidad del procedimiento de agrupamiento y ofreció ahorros de recursos de BBU bastante cercanos en comparación con FFD (Primer ajuste de cribado).

Referente a la gestión de interferencia, Hossain, Rasti, Tabassum, \& Abdelnasser (2014) en su investigación determinó que los sistemas 5G adoptan una arquitectura de múltiples niveles que consiste en macrocélulas, diferentes tipos de celdas pequeñas con licencia, relés y redes de dispositivo a dispositivo (D2D) para servir a los usuarios con diferentes requisitos de calidad de 
servicio (QoS) en un espectro y eficiencia energética, por lo cual el autor utilizó el método de Control de potencia oportunista (OPC) que asigna altos niveles de potencia a usuarios con buenos canales y muy baja potencia a usuarios con canales pobres, concluyendo que en este algoritmo, una pequeña diferencia en las ganancias de ruta entre dos usuarios puede conducir a una gran diferencia en su rendimiento real.

$\mathrm{Xu}, \mathrm{Li}, \&$ Yang (2018) estudiaron la asignación de recursos inteligentes basada en algoritmos genéticos mejorados en redes ultra densas $5 \mathrm{G}$, por lo cual formularon un problema de optimización de objetivos múltiples (MOOP), proponiendo una versión mejorada del enfoque inteligente basado en el algoritmo genético de clasificación no dominado II (NSGA-II) que permite a los usuarios de células pequeñas optimizar su enlace descendente de Eficiencia Energética (EE) y Eficiencia del Espectro (SE) mediante la asignación conjunta de energía de transmisión y bloques de recursos. En lugar de utilizar el método tradicional de suma ponderada que no puede obtener el EE y el SE óptimos simultáneamente, se propone un algoritmo de asignación de recursos basado en NSGA-II mejorado, este algoritmo propuesto produce ganancias de rendimiento significativas en comparación con la búsqueda exhaustiva existente.

De igual forma, UI Najam, et al. (2016) propusieron un algoritmo de selección de red efectivo para redes heterogéneas $5 \mathrm{G}$ que puede elegir eficientemente la red con velocidad de datos garantizada y rendimiento del usuario. Se resolvió el problema de optimización con la Optimización de enjambre de partículas (PSO) y el Algoritmo Genético (GA) modificado para encontrar una solución casi óptima. Los resultados de la simulación mostraron que el GA modificado supera al PSO y logra un mayor valor de aptitud con menos iteraciones en términos de reducción de interferencia y requisito de precio de $\mathrm{SU}$, así también en la investigación denominada "Colocación de nodo de servicio Pico basado en lógica difusa para red ultradensa 5G" se propuso un método de colocación de estación base de nodo evolucionado (eNB) con base difusa para cumplir con las demandas de cobertura, sin perturbar los eNB macro existentes, y mientras se satisfacen las restricciones de interferencia y presupuesto (Murugadass, 2019).

Las redes $5 \mathrm{G}$, al poseer un alto contenido de ancho de banda con velocidades superiores a $10 \mathrm{~Gb} /$ $\mathrm{s}$, varios niveles de movilidad y soluciones energéticas y rentables con el aumento de la inteligencia del mundo inalámbrico deben satisfacer todas las necesidades de los usuarios, lo que es llamado como calidad de experiencia, por lo cual, Al-Maitah, Semenova, Semenov, Kulakov, \& Kucheruk (2018) propuso una metodología para desarrollar un controlador combinando un sistema difuso con una red neuronal artificial y un algoritmo genético para la admisión de llamadas en redes 5G. El enfoque propuesto puede aumentar la calidad del servicio al disminuir la probabilidad de bloqueo de llamadas de las nuevas llamadas entrantes en una red. El rendimiento del control de admisión propuesto se evalúa mediante simulación por computadora. El controlador difuso propuesto se convertirá en un sistema de inferencia neurofuzzy adaptativo (ANFIS), adecuado para operar en condiciones inciertas, es por ello que se concluye que para actualizar la base de reglas se debe utilizar un algoritmo genético. 
En concordancia con lo expuesto, se añade que el usuario no evalúa únicamente el elemento de red individual, es decir considera el rendimiento general del sistema, el precio del servicio, la calidad percibida del contenido y la facilidad de uso de una aplicación, es decir nuevamente el QoE o calidad de experiencia, por lo cual Pierucci (2015), propuso un método eficiente de estimación de la calidad de la experiencia (QoE) adaptado para sistemas 5G basado en el enfoque de red neuronal $(\mathrm{NN})$, en donde menciona que el uso de NN también puede garantizar la replicabilidad de las estimaciones de QoE independientemente de la participación del usuario, que es el principal inconveniente en las pruebas subjetivas basadas en puntuación de opinión media (MOS). A partir de las consideraciones anteriores, el uso de redes neuronales se convierte en una propuesta adecuada para una estimación adaptativa y una clasificación automática de la calidad percibida por el usuario en los sistemas 5G, por lo que concluyó que solo una combinación de mejoras junto con dispositivos de radio cognitivos nuevos y eficientes enriquecerá la satisfacción general del usuario a un alto nivel en los sistemas 5G.

Además, Liu, Chuai, \& Gao (2018) propuso un diseño de celda virtual basado en lógica difusa para equilibrar las velocidades de datos y la sobrecarga de recursos a fin de mejorar la aplicabilidad de la celda virtual. En el diseño se consideró un escenario práctico de UDN con información de estado de canal imperfecto (CSI) y se lo modeló mediante la teoría de la geometría estocástica. De acuerdo a ello se propuso un algoritmo de selección de estrategia de activación basada en lógica difusa (FASS) para elegir racionalmente el umbral de activación de celda virtual y el esquema de asignación de recursos bajo diferentes cargas de tráfico y requisitos de calidad de servicio (QoS). Así también, para abordar los desafíos de transferencia y proporcionar una alta calidad de servicio al usuario Liu, Foong, Zhang, \& Li (2019) propuso un esquema de transferencia, que integra las ventajas de la lógica difusa y los algoritmos de decisión de atributos múltiples (MADM) para garantizar que el proceso de transferencia se active en el momento adecuado y la conexión se cambie a la BS adyacente óptima. Para mejorar aún más el rendimiento del esquema propuesto, también adopta la técnica de agrupamiento sustractivo mediante el uso de datos históricos para definir las funciones óptimas de membresía dentro del sistema difuso.

Zhang et al., (2017) manifiesta que para que las redes 5G pueda proporcionar servicios adaptados a las demandas específicas de QoS de los usuarios se puede establecer una estrategia de segmentación de servicios centrada en el usuario que considera diferentes requisitos de QoS en función de la red definida por software (SDN), por lo cual diseñó un algoritmo genético para optimizar la gestión de recursos de radio virtualizada basada en la agrupación de recursos, dicho rendimiento prometedor de las redes $5 \mathrm{G}$ basadas en el corte de red se demostró a través de simulaciones por computadora. Dentro del parámetro de la calidad de servicio (Lounis, Alilat, \& Nazin (2018) basó su investigación en un modelo de red neuronal de QoE para la estimación de transmisión de video a través de la red 5G, en el cual se presentaron aspectos específicos considerados importantes en la evolución de $4 \mathrm{G}$ a $5 \mathrm{G}$ en términos de gestión del tráfico y una solución para estimar este QoE en este nuevo contexto. Además se adoptó un enfoque basado en la red neuronal $(\mathrm{NN})$ para estimar los parámetros de calidad de experiencia demostrando que las 
redes neuronales se han utilizado con éxito en muchos dominios donde era difícil obtener un modelo analítico exacto del sistema, como es el caso de la red 5G.

Dichas investigaciones se complementan con el trabajo desarrollado por Zhao, Yang, Guo, Peng, $\&$ Zhang (2019), quienes diseñaron un sistema basado en el aprendizaje profundo autoadaptativo para la detección de anomalías en redes 5G, dichos autores presentaron un método preciso de localización de fallas basado en una red de evolución neuronal profunda en redes ópticas. Los experimentos indicaron que el método propuesto mejora la precisión de la ubicación de la falla cuando se enfrenta a conjuntos de alarmas a gran escala. Así también You, Zhang, Tan, Jin, \& Wu (2019) proporcionaron una visión general de las direcciones de la inteligencia artificial para tecnologías 5G, motivo por el cual establecieron un paradigmas de diseño que incluyen optimización de red 5G, asignación óptima de recursos, aceleración unificada de capa física 5G, optimización conjunta de capa física de extremo a extremo, etc.

En referencia a esta investigación se añade el estudio de Khan, Abolhasan, Ni, Lipman, \& Jamalipour (2019) en el cual se propuso un enfoque de algoritmo genético guiado por lógica difusa híbrida (H FLGA) para el controlador de red definido por software, que tuvo como finalidad resolver un problema de optimización de recursos multipropósito para VANETs 5G. Este enfoque se centra en ayudar a los proveedores de servicios de red a implementar una infraestructura de red centrada en el cliente, dependiendo de las necesidades dinámicas de los usuarios de los usuarios.

Así tambien, Xu, Zhang, Dai, Hou, \& Tao (2014) se investigó la división de servicios orientados a dispositivos móviles con diferentes clasificaciones de calidad de servicio (QoS) y se introduce la gestión de recursos de radio virtualizada (VRRM) basada en agrupación de recursos en la Arquitectura de red sin marco (FNA) basado en Red definida por software (SDN). Basado en la característica de recursos limitados dentro de las redes móviles, la solución de optimización de VRRM se deduce con Algoritmo Genético (GA). Entre las principales contribuciones se destaca: La incorporación de SDN en redes móviles con virtualización de red, realización de la división de servicios en redes móviles, Realización de una red de prueba basada en MiniNet para SDN con FNA. De igual manera Sun, Gong, Rong, \& Lu (2015) propusieron un modelo inteligente basado en una red definida por software (SDN) que puede administrar eficientemente la infraestructura y los recursos heterogéneos de la red 5G, de acuerdo al análisis de las estrategias de SDN inteligente con respecto a cuatro aspectos de las redes 5G: predicción de tráfico, equilibrio de carga, predicción de densidad de usuario y asignación de recursos de radio se utilizó la asignación conjunta de recursos basada en algoritmos genéticos (GA), dicho GA proporciona un medio eficaz para resolver este problema de optimización no lineal. En realidad, el GA presenta una capacidad de búsqueda global significativa y robustez en la resolución de problemas en las redes de quinta generación.

A pesar de los avances y estudios realizados acerca del uso de las técnicas de soft computing para solucionar problemas de infraestructura de las redes 5G, Albarracin \& Puerto (2017) en su estudio denominado "Sistemas difusos: un enfoque para redes $5 \mathrm{G}$ bajo el paradigma de red definido por 
software" indican que la implementación de sistemas difusos para la toma de decisiones en los diversos sistemas de telecomunicaciones no ha sido muy explotada; pues solo los sistemas difusos tipo I se han aplicado en los desarrollos, enfatizando dos tipos de sistemas difusos: el sistema difuso tipo Mamdani y el de tipo Sugeno. Esta imagen actual del desarrollo tecnológico proporciona un amplio espectro de oportunidades para el desarrollo e integración de sistemas difusos en sistemas emergentes de telecomunicaciones móviles.

Kaloxylos, Barmpounakis, \& Alonistioti (2014) describieron un nuevo esquema de transferencia de criterios múltiples, además de brindar detalles sobre las soluciones para adquirir la información contextual necesaria y describir el algoritmo para seleccionar las Tecnologías de acceso de radio (RAT) más apropiadas. La solución planteada se basó en el uso de controladores de lógica difusa para combinar diversas entradas (como la movilidad de un usuario, la carga de las estaciones base candidatas, entre otras.). Se concluyó que el uso de lógica difusa ayuda a guiar los terminales hacia eNBs $(\mathrm{H})$ o APs Wi-Fi descargados para usuarios estáticos o de baja movilidad, así también mejora la eficiencia del sistema en general.

En la investigación "Control de velocidad de flujo difuso adaptativo considerando modelado de tráfico multifractal y comunicaciones 5G", los autores Cardoso \& Vieira (2019) realizaron un esquema predictivo de control de flujo generalizado OBF (funciones de base ortonormal) para el enlace descendente 5G derivando una expresión para la tasa de control óptima de las fuentes de tráfico considerando la minimización del retraso de datos y una tasa de tráfico mínima para los usuarios.

El modelo adaptativo GOBF-Fuzzy se aplica para predecir el comportamiento de las colas en los sistemas 5G iniciales. Con esta investigación se puede añadir que existen varias propuestas de esquemas de control en la literatura que se enfocan a las redes 5G, tal es el caso de Rashad (2019), quien diseñó un esquema de predicción de transferencia y selección de red objetivo para redes 5GIoT. Para la condición de activación de VHO, se aplica la red de avance de alimentación multicapa (MFNN) que predecirá la movilidad del usuario en función de los parámetros de distancia, RSS, velocidad móvil y dirección, y para la selección de celda objetivo, el modelo de decisión difuso se aplica en función de las métricas de nivel de red, como la carga de tráfico, la latencia de transferencia, la energía de la batería y las métricas de nivel de usuario, como la seguridad y el costo.

Zapata, et al., (2018) plantearon una forma de reducir el coste de las redes ultradensas, la cual consiste en el apagado de un subconjunto de Estaciones Base de tal forma que se minimice el consumo energético y al mismo tiempo se eleve la capacidad de la misma, proponiendo un nuevo operador de búsqueda local llamado CSO, que se ha integrado dentro de dos algoritmos del dominio, uno que es conocido como NSGA-II, y el otro reciente denominado MOCell, sus versiones híbridas han logrado aproximaciones al frente de Pareto del problema con una mejora notable en el indicador hypervolumen (HV). 
El rendimiento de la red es un factor imprescindible para que los clientes seleccionen un operador de red móvil (MNO), puesto que los clientes exigen movilidad y servicios sin interrupciones con una pérdida mínima de paquetes, por lo que la comunicación de dispositivo a dispositivo (D2D) es una de las soluciones clave de habilitación de la quinta generación (5G), por ello en el trabajo realizado por Subramani \& Kumaravelu (2018) se propuso una estimación de necesidad de traspaso basada en lógica difusa de tres etapas y un esquema de selección de objetivos para redes heterogéneas generales, con lo cual se pudo demostrar que el PLR, el número de traspasos ejecutados y los rendimientos del esquema propuesto son superiores a los esquemas de toma de decisiones de atributos múltiples (MADM) convencionales y difusos.

Mientras que, Abdelbari \& Haci (2019) propucieron un novedoso esquema de programación de usuarios basado en la lógica difusa llamado "Evaluación de programación de usuarios basada en difusa (FUSE)" para redes inalámbricas ultra densas, el mismo que tuvo como objetivo superar un inconveniente de los esquemas de programación de vanguardia: Ración Máxima de Señal a Ruido (Max-SNR), Equidad Proporcional (PF), Equidad Exponencial / Proporcional (EXP / PF) y Retraso Ponderado más grande Modificado Primero (M-LWDF) al proporcionar un mejor equilibrio entre los parámetros relacionados con el retraso y el rendimiento en la toma de decisiones de programación. Los resultados de rendimiento demuestran que el esquema propuesto supera a EXP / PF y M LWDF.

De acuerdo a lo expuesto se evidencia el desarrollo de investigaciones en donde se proponen algoritmos de selección de red efectivo para redes heterogéneas $5 \mathrm{G}$ que permitan elegir eficientemente la red con velocidad de datos esperada y rendimiento del usuario, tal es el caso de Lynch, Saber, \& Kucer (2019) cuya investigación se basa en la automatización del proceso de diseño de asignación de enlaces, a través de la aplicación novedosa de algoritmos evolutivos, considerando que los programadores evolucionados aumentan las tasas de enlace descendente en más de un $150 \%$ para los usuarios con peor rendimiento, en relación con una línea base de enlace único.

Los hallazgos de este estudio muestran claramente que las redes 5G tienen como finalidad mejorar las velocidades de transmisión de los datos, proporcionar excelente rendimiento de extremo a extremo, brindar una mayor cobertura del usuario y áreas concurridas con menor latencia, consumo de energía y costo por transferencia de información, para lo cual, estos sistemas pueden adoptar una arquitectura de varios niveles que consiste en macrocélulas, diferentes tipos de celdas pequeñas con licencia, relés y redes de dispositivo a dispositivo (D2D) para servir a los usuarios con diferentes requisitos de calidad de servicio en un espectro y de manera eficiente de la energía. Pero a pesar de ello, la tecnología 5G no han definido de manera clara la forma en la que se ofrecerá los servicios de telecomunicaciones, por tal motivo se presentan varios inconvenientes a la hora de la implementación del servicio, pues como se indica Zapata, et al., (2018) para la implementación de las redes $5 \mathrm{G}$ se requiere de miles de Estaciones Base (BSs) por $\mathrm{Km}^{2}$, lo cual representa uno de los principales conflictos para el diseño de este tipo de redes que tiene como finalidad tener un $90 \%$ 
menos de consumo energético que la cuarta generación actual. Ante esta problemática se puedo evidenciar que las técnicas de soft computing sirven de gran ayuda para mejorar la calidad del servicio de la tecnología $5 \mathrm{G}$, pues se basan en el diseño de sistemas inteligentes capaces de manejar de manera oportuna la información incierta, tal es el caso de la lógica difusa que se utiliza principalmente para limitar el consumo de energía en la infraestructura de las redes 5G.

\section{Conclusiones.}

- En el presente artículo se realizó un análisis de diversos documentos de revisión acerca de las técnicas de soft computing aplicadas a la solución de problemas de planificación de las redes 5G, en los cual se ha evidenciado la utilización de la lógica difusa, para limitar el consumo de energía en las infraestructuras de red 5G, lo que representa uno de los principales conflictos para el diseño de este tipo de redes.

- Con la investigación se pudo determinar que el planteamiento de controlador combinando un sistema difuso con una red neuronal artificial y un algoritmo genético que son técnicas de soft computing, para la admisión de llamadas en redes 5G tienen como finalidad incrementar la calidad del servicio al disminuir la probabilidad de bloqueo de llamadas.

- Entre las limitantes más importantes para la implementación de las redes de quinta generación señaladas en la mayoría de los artículos de revisión se encuentra el consumo energético, que se deriva de la integración de distintas interfaces y del incremento de requerimientos en lo que se refiere al procesamiento de señales, encontrando como solución el planteamiento del apagado de celdas en el contexto de los despliegues ultradensos de estaciones base, pues existen ocasiones en que dentro de una celda no se encuentran dispositivos a los cuales proveer un servicio, razón por la cual no se necesita que la celda posea la una máxima potencia, sino que se puede hibernar y as $\{i$ ahorrar el consumo de energía.

\section{Referencias Bibliográficas.}

Abdelbari, A., \& Haci, H. (noviembre de 2019). Fuzzy Logic-Based User Scheduling Scheme for 5G Wireless Networks and Beyond. Springer Link.

Agredo, G., Jojoa, P., \& Almenar, V. (julio-diciembre de 2015). Sistemas MIMO con un elevado número de antenas: clave para la 5G inalámbrica. Entramado, 11(2), 250-261.

Albarracin, L., \& Puerto, G. (2017). Fuzzy Systems: An Approach to 5G Networks under the Software Defined. Revista Científica, 31(1), 96-110.

Al-Maitah, M., Semenova, O., Semenov, A., Kulakov, P., \& Kucheruk, V. (octubre de 2018). A Hybrid Approach to Call Admission Control in 5G Networks. Hindawi: Advances in Fuzzy Systems, 1-7. 
Angulo, K., Gil, D., \& Salcedo, O. (mayo de 2017). Optimización utilizando lógica difusa de dispositivo de análisis de componentes químicos de ingredientes naturales basados en el internet de las cosas IoT. Revista Científica, 30(3), 207-223.

Atayero, A., \& Luka, M. (mayo de 2012). Applications of Soft Computing in Mobile and Wireless Communications. International Journal of Computer Applications, 45(22), 48-52.

Cardoso, A., \& Vieira, F. (2019). Adaptive fuzzy flow rate control considering multifractal traffic modeling and 5G communications. Journals, 14(11).

Castañeda, E., Garmendia, L., \& Santos, M. (2011). Algoritmos Genéticos Difusos: Una Aproximación Práctica para la Creatividad Computacional. Segundo Congreso Internacional de Matemáticas en la Ingeniería y la Arquitectura, 279-290.

Chabbouh, O., Ben, S., \& Choukair, Z. A. (2018). A Two-stage RRH Clustering Mechanism in 5G Heterogeneous C-RAN. 5th International Workshop on ADVANCEs in ICT Infrastructures.

Crespo, J., Peña, E., Pascual, V., \& Fustiel, J. (2016). Elección entre una metodología ágil y tradicional basado en técnicas de soft computing. Revista Cubana de Ciencias Informáticas, 10.

Demestichas, K., Adamopoulou, E., \& Choras, M. (abril de 2017). 5G Communications: Energy Efficiency. Hindawi, 3.

Fundacio Universitat Empresa. (2019). Estudio sobre la planificación, el impacto y la cobertura de redes $4 G$ y $5 G$ de comunicaciones móviles. Recuperado el 24 de septiembre de 2019, de Ministerio de Ciencias, Innovación y Universidades: https://fueib.org/es/investigadors/65/otri/catalogo/5/741/servicio/estudio-sobre-laplanificacion-el-impacto-y-la-cobertura-de-redes-4g-y-5g-de-comunicaciones-moviles

Gjokaj, V., Doroshewitz, J., Nanzer, J., \& Chahal, P. (2017). A Design Study of 5G Antennas Optimized Using Genetic Algorithms. 2017 IEEE 67th Electronic Components and Technology Conference.

González, C. (2011). Lógica Difusa: Una introducción práctica. La Mancha: Universidad de Castilla.

González, J., \& Salamanca, Ó. (enero-junio de 2016). El camino hacia la tecnología 5G. Revista Electrónica de Estudios Telemáticos, 15(1), 27-47.

Hossain, E., Rasti, M., Tabassum, H., \& Abdelnasser, A. (2014). Evolution Towards 5G Multi-tier Cellular Wireless Networks: An Interference Management Perspective. arXiv, 2, 1-10. 
Kaloxylos, A., Barmpounakis, S., \& Alonistioti, N. (2014). An efficient RAT selection mechanism for 5G cellular networks. (Mobile and Wireless Communications Enablers for the 2020 Information Society.

Khan, A., Abolhasan, M., Ni, W., Lipman, J., \& Jamalipour, A. (2019). A Hybrid-Fuzzy Logic Guided Genetic Algorithm (H-FLGA) Approach for Resource Optimization in 5G VANETs. IEEEexplore, 68(7), 6964 - 6974.

Kumar, P., \& Mahajan, A. (2009). Soft Computing Techniques for the Control of an Active Power Filter. IEEE Transactions on Power Delivery, 24(1), 452-461.

Kumar, S., Kumar, A., Das, B., \& Burnwal, A. (2013). On Soft Computing Techniques in varios areas. Computer Science \& Information Technology (CS \& IT), 59-68.

Liu, Q., Chuai, G., \& Gao, W. (2018). uzzy Logic-based Virtual Cell Design in Ultra-Dense Networks. Wireless Com Network 2018, 87.

Liu, Q., Foong, C., Zhang, S., \& Li, L. (septiembre de 2019). A fuzzy-clustering based approach for MADM handover in $5 \mathrm{G}$ ultra-dense networks. Springer.

López, M., Guerra, M., \& Izaquirre, S. (junio de 2017). Profundización en la introducción de las redes móviles 5G. Revista Técnica de la Empresa de Telecomunicaciones de Cuba S.A., $14,36-45$.

Lounis, A., Alilat, F., \& Nazin, A. (2018). Neural Network Model of QoE for Estimation Video Streaming over 5G network. 6th International Workshop on ADVANCEs in ICT Infrastructures and Services, 21-27.

Lynch, D., Saber, T., \& Kucera, S. (2019). Evolutionary learning of link allocation algorithms for 5G heterogeneous wireless communications networks. Proceedings of the Genetic and Evolutionary Computation Conference, 1258-1265.

Maldonado, S. (2013). Técnicas de soft-computing para el desarrollo de redes de acceso móvil con control de polución electromagnética. Madrid: Universidad de Alcalá.

Martín, A. (2015). Integración de técnicas de soft-computing $n$ la planificación basada en conocimiento de tareas de desarrollo software. La Mancha, España: Universidad de Castilla.

Ministerio de Tecnologías de la Información y las Comunicaciones de Colombia. (2019). Plan 5g: El futuro digital es de tods. Colombia.

Murugadass, A. (2019). A Fuzzy Logic Based Pico Serving Node Placement for 5G Ultra Dense Networks. International Journal of Advanced Science and Technology, 28(19), 108-115. 
Panda, M., \& Patra, M. (2013). Soft: Computing:Concepts and Techniques (Primera ed.). New Delhi, Boston, USA: University Science Press.

Peñaranda, C. (2015). Aplicación de Técnicas Soft Computing y Heurísticas para la identificación y clasificación de la información empleada por un recomendador de recetas. Valencia: Universitat Politecnica de Valencia.

Pierucci, L. (2015). The Quality of experience perspective toward 5G technology. IEE Wireless Comunnications, 22(4), 10-16.

Popovski, P., Trillingsgaard, Kasper, Simeone, O., \& Durisi, G. (2018). 5G Wireless Network Slicing for eMBB, URLLC, and mMTC: A Communication-Theoretic View. Open Access Journal, 6.

Premnath, K. N., Srinivasan, R., \& Elijah, B. (septiembre de 2014). Magnetic Field Model (MFM) in Soft Computing and parallelization techniques for Self Organizing Networks (SON) in Telecommunications. International Journal of Energy Optimization and Engineering, 3(3), $57-71$.

Ramírez, J., Sarmiento, H., \& López, J. (2018). Diagnóstico de fallas en procesos industriales mediante inteligencia artificial. Revista Espacios, 39(24), 12.

Rashad, C. (2019). Fuzzy-Neural based Cost Effective Handover Prediction Technique for 5G-IoT networks. International Journal of Innovative Technology and Exploring Engineering, 9, 191-197.

Rodríguez, A., \& Pérez, A. (2017). Métodos científicos de indagación y de construcción del conocimiento. Revista Escuela de Administración de Negocios(82), 1-26.

Sachan, R., Jong Choi, T., \& Wook, C. (abril de 2016). A Genetic Algorithm with Location Intelligence Method for Energy Optimization in 5G Wireless Networks. Hindawi, 1-9.

Sanou, B. (2018). Sentando las bases para la 5G: Oportunidades y desafíos. Desarrollo de las Telecomunicaciones de la UIT.

Santos, M., \& Miranda, E. (junio de 2012). Aplicación de la lógica difusa en el ámbito de las energías renovables. Revista Elementos, 2(1), 102-114.

Subramani, M., \& Kumaravelu, V. (2018). A Three-Stage Fuzzy-Logic-Based Handover Necessity Estimation and Target Network Selection Scheme for Next Generation Heterogeneous Networks. Journal of Circuits.

Sun, J., shi, W., Yang, Z., \& Yang, J. (2019). Behavioral Modeling and Linearization of Wideband RF Power Amplifiers Using BiLSTM Networks for 5G Wireless Systems. IEEE Transactions on Vehicular Technology, 1(1). 
Sun, S., Gong, L., Rong, B., \& Lu, K. (2015). An Intelligent SDN Framework for 5G Heterogeneous Networks. IEEE Communications Magazine, 53(11), 142-147.

UI Najam, H., Ejaz, W., Ejaz, N., Kim, H., Anpalagan, A., \& Jo, M. (2016). Network Selection and Channel Allocation forSpectrum Sharing in 5G Heterogeneous Networks. IEEE Access, 4, 980-992.

Veslin, E. (2013). Aplicación de algoritmos genéticos en problemas de Ingeniería . Revista de Investigación, Innovación e Ingeniería, 10-29.

Xu, S., Li, R., \& Yang, Q. (2018). Improved Genetic Algorithm Based Intelligent Resource Allocation in 5G Ultra Dense Networks. 2018 IEEE Wireless Communications and Networking Conference (WCNC).

Xu, X., Zhang, H., Dai, X., Hou, Y., \& Tao, X. Z. (2014). SON Based Next Generation Mobile Network With Service Slicing and Trials. China Communications, 11(2), 65-77.

You, X., Zhang, C., Tan, X., Jin, S., \& Wu, H. (2019). AI for 5G: research directions and paradigms. Sci China Inf Sci, 62(2).

Zapata, P., Luna, F., Valenzuela, J., Mora, A., \& Padilla, P. (2018). Metaheurísticas híbridas para el problema del apagado de celdas en redes 5G. XIII Congreso Espanol en Metaheurísticas y Algoritmos Evolutivos y Bioinspirados, 18, 665-670.

Zhang, H., Liu, N., Chu, X., Long, K., Aghvami, A., \& Leung, V. (2017). Red segmentada de redes 5G y redes móviles futuras: movilidad, gestión de recursos y desafíos. IEEE Communications Magazine, 55(8), 138-145.

Zhao, X., Yang, H., Guo, H., Peng, T., \& Zhang, J. (2019). Accurate Fault Location based on Deep Neural Evolution Network in Optical Networks for 5G and Beyond. ptical Fiber Communication Conference (OFC) 2019.

\section{【 Ciencia}




\section{PARA CITAR EL ARTÍCULO INDEXADO.}

Vanessa Alexandra, V. N. (2020). Análisis del estado de arte de Técnicas de Soft Computing aplicadas a problemas de planificación de red en 5G. ConcienciaDigital, 3(2.2), 60-79. https://doi.org/10.33262/concienciadigital.v3i2.2.1246

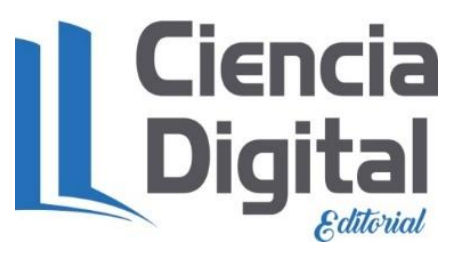

El artículo que se publica es de exclusiva responsabilidad de los autores y no necesariamente reflejan el pensamiento de la Revista Ciencia Digital.

El artículo queda en propiedad de la revista y, por tanto, su publicación parcial y/o total en otro medio tiene que ser autorizado por el director de la Revista Ciencia Digital.
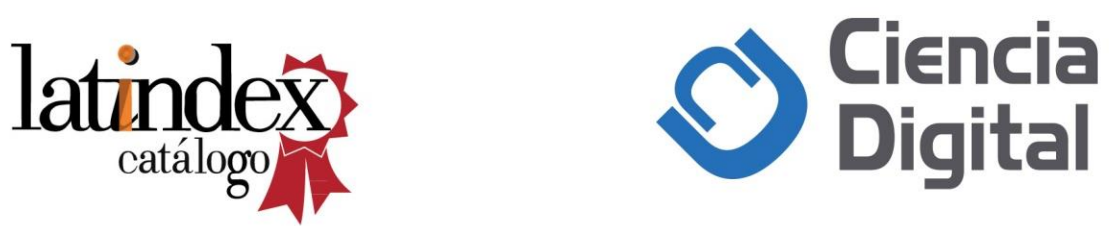